\title{
UAV Air-to-Ground Channel Characterization for mmWave Systems
}

\author{
Wahab Khawaja, Ozgur Ozdemir, and Ismail Guvenc \\ Department of Electrical and Computer Engineering, North Carolina State University, Raleigh, NC \\ Email: \{wkhawaj, oozdemi, iguvenc\}@ncsu.edu
}

\section{प}

Abstract-Communication at mmWave bands carries critical importance for $5 \mathrm{G}$ wireless networks. In this paper, we study the characterization of mmWave air-to-ground (AG) channels for unmanned aerial vehicle (UAV) communications. In particular, we use ray tracing simulations using Remcom Wireless InSite software to study the behavior of AG mmWave bands at two different frequencies: $28 \mathrm{GHz}$ and $60 \mathrm{GHz}$. Received signal strength (RSS) and root mean square delay spread (RMS-DS) of multipath components (MPCs) are analyzed for different UAV heights considering four different environments: urban, suburban, rural, and over sea. It is observed that the RSS mostly follows the two ray propagation model along the UAV flight path for higher altitudes. This two ray propagation model is affected by the presence of high rise scatterers in urban scenario. Moreover, we present details of a universal serial radio peripheral (USRP) based channel sounder that can be used for AG channel measurements for mmWave (60 GHz) UAV communications.

Index Terms-28 GHz, $60 \mathrm{GHz}$, channel model, drone, mmWave, ray tracing, unmanned aerial vehicle (UAV), USRP.

\section{INTRODUCTION}

Unmanned aerial vehicles (UAVs) are envisioned to support numerous applications in 5G wireless networks [1], [2]. They can serve as mobile hot spots in congested areas [3], help in improving the quality of experience of wireless users by serving as a content cache [4], and support communication needs of first responders at times/locations where most needed [5]. Due to their agile, three dimensional mobility in free space, UAVs can easily move from one place to another in order to provide on demand communication support, can change their locations to avoid blockage/shadowing, and can help in data relaying/ferrying applications.

Use of mmWave bands can be very promising for UAV communications to sustain data rate demands for high throughput mobile applications. In particular, UAVs can maintain line-ofsight (LOS) connectivity (or at least a reasonable non-LOS (NLOS) link) with a desired user by hovering at a favorable location [3], which is crucial in maintaining a good link quality at mmWave bands due to large path loss. A number of licensed/unlicensed mmWave bands are potentially available for use in UAV communications: see e.g. FCC's spectrum

This work has been supported in part by the National Science Foundation under the grants CNS-1618692 and CNS-1453678, and W. Khawaja has been supported via a Fulbright scholarship. frontiers report and order for bands above $24 \mathrm{GHz}$ [6], and FCC's amendment for use of 57-60 GHz unlicensed band for outdoor communications [7]. In this paper, we consider the $28 \mathrm{GHz}$ licensed bands and the $60 \mathrm{GHz}$ unlicensed bands for their potential use in mmWave UAV communications. While propagation characteristics for both mmWave bands have been studied for urban scenarios in the literature [8][11], and air-to-ground (AG) propagation measurements are recently reported for the UWB spectrum below $6 \mathrm{GHz}$ [12], to our best knowledge characteristics of the AG UAV links have not been studied yet for the mmWave bands.

In this paper, we analyze AG propagation characteristics for the $28 \mathrm{GHz}$ and $60 \mathrm{GHz}$ mmWave bands using ray tracing simulations. The analysis is carried out considering four different environments: urban, suburban, rural, and over sea. Each scenario has different number of scatterers at different heights. The transmitter (ground station (GS)) location is at a fixed position, while the UAV that carries the receiver node flies on a linear path. A single, omni-directional antenna is used for both the transmitter and the receiver. The simulations are run for different UAV heights for each scenario, considering both $28 \mathrm{GHz}$ and $60 \mathrm{GHz}$ bands. The velocity of the UAV is fixed at $15 \mathrm{~m} / \mathrm{s}$. Due to high path loss at mmWave bands, flight distance was limited to $2 \mathrm{~km}$.

Based on the simulation results it is observed that the received signal strength (RSS) reasonably follows the two ray propagation model [13], [14]. On the other hand, the two ray propagation model holds better at shorter transmitter-receiver separations; after some distance, only the dominant component is the main contributor to the RSS, and reflected path from the ground is negligible. The reflections and diffractions from the scatterers in the environment introduce fluctuations in the RSS that are dependent on the density and height of the scatterers. In case of large number of high-rise scatterers such as in the urban environment, there are rapid fluctuations in the RSS, and the two ray propagation model cannot be directly applied. The effect of scatterers is higher at $28 \mathrm{GHz}$ compared to $60 \mathrm{GHz}$ as can be observed from the root mean square delay spread (RMS-DS) of multipath components (MPCs).

In addition to ray tracing simulations, we have built an AG mmWave channel sounder using universal serial radio peripheral (USRP) X310 software defined radios (SDRs), and frequency up-converters from Pasternack. The in-phase (I) and 


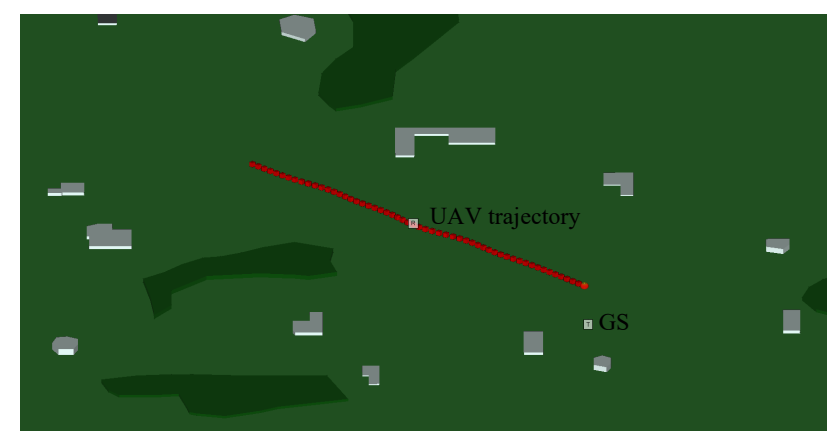

(a)

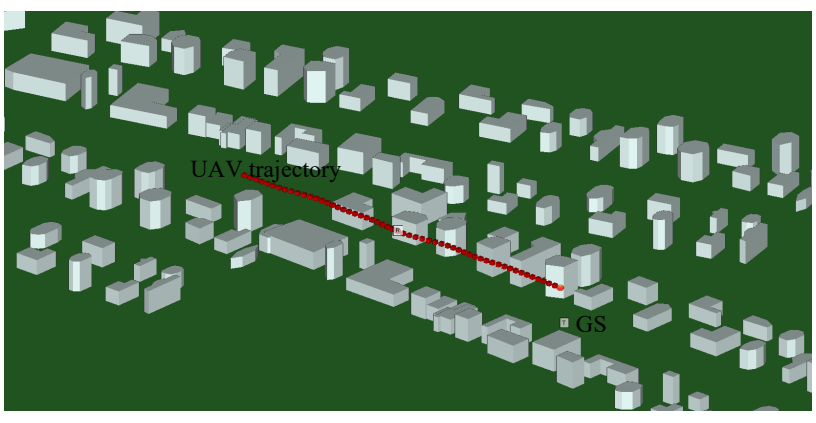

(b)

Fig. 1. UAV AG propagation scenarios simulated using Remcom Wireless InSite software. Transmitter is located on the ground, and UAV with the onboard receiver follows the illustrated trajectory. (a) Sub-urban scenario, (b) Urban scenario.

quadrature $(\mathrm{Q})$ components from the USRP X310 are upconverted to 57-64 GHz unlicensed band. A basic channel sounder with frequency offset correction is built using GNU radio, and associated block diagrams for channel sounding are provided. Due to compact size of the setup, transmitter/receiver can be easily air lifted with moderate payload capacity UAVs. Our future work includes AG measurements with DJI S-1000 UAV using this sounder setup.

The rest of this paper is organized as follows. In Section II ray tracing simulations for AG channel characterization in mmWave bands is described. Section $\amalg$ introduces the details of the SDR based channel sounder at the $60 \mathrm{GHz}$ band. Finally, the last section provides some concluding remarks.

\section{RAY TRACING SimUlations FOR MMWAVE UAVS}

Ray tracing can provide a deterministic way of characterizing the mmWave channel in different scenarios. Due to challenges involved in AG channel measurements at mmWave bands using UAVs, ray tracing offers a convenient alternative way of evaluating the channel behavior. In this section, we use the Remcom Wireless InSite ray tracing software, and imitate the real time motion of the UAV over a given trajectory. We will first describe the different ray tracing scenarios considered, followed by RSS and RMS-DS results.
TABLE I

RAY TRACING SIMULATION PARAMETERS.

\begin{tabular}{|c|c|c|}
\hline Scenario & Building height $(\mathbf{m})$ & Number of buildings \\
\hline Over sea & - & - \\
\hline Rural & $4-8$ & 10 \\
\hline Suburban & $4-30$ & 20 \\
\hline Urban & $70-180$ & 100 \\
\hline
\end{tabular}

\section{A. Ray Tracing Scenarios}

We consider four different scenarios for ray tracing simulations: urban, suburban, rural, and over sea. Three key factors for AG ray tracing simulations in a given scenario are the number, material, and height of the scatterers in the environment. The largest scatterers in AG propagation are the buildings. The effect of mobile vehicles on road as potential scatterers is negligible in AG propagation. We considered different number of buildings at different heights in the simulations as summarized in Table II The urban scenario has the highest density and height of randomly shaped buildings followed by suburban and rural. Additionally, there is scattered foliage introduced in the suburban and rural scenarios. The material of the buildings is frequency sensitive concrete at $28 \mathrm{GHz}$ and $60 \mathrm{GHz}$ for all the scenarios except the simulations over the sea. For simplicity, we considered the building material of all the buildings as concrete. For all the land based simulations, a dry ground is considered. Fig. 1 1 shows the suburban and urban scenarios with the GS on the ground, and UAV trajectory at is shown at a height of 150 meters above the ground. In case of over sea scenario, a 10 meter layer of sea water is considered covering a typical terrain. A couple of metallic ships on the surface of the sea water are introduced as scatterers.

The characteristics of the simulation set up are as follows. The dimensions of the terrain are $10 \mathrm{~km}$ by $10 \mathrm{~km}$. The height of the transmitter is $2 \mathrm{~m}$ and is fixed on ground. The UAV heights are $2 \mathrm{~m}$ (imitating a mobile vehicle on the ground), $50 \mathrm{~m}, 100 \mathrm{~m}, 150 \mathrm{~m}$ above the ground, with a velocity of $15 \mathrm{~m} / \mathrm{s}$ for all cases. The length of the UAV trajectory is around $2 \mathrm{~km}$. Half-wave dipole antennas with vertical polarization are used on both transmitter and the receiver. A sinusoid is used to sound the channel at two center frequencies of $28 \mathrm{GHz}$ and $60 \mathrm{GHz}$. The transmit power is set at $30 \mathrm{dBm}$.

\section{B. Ray Tracing Results for the RSS}

The RSS as a function of the distance between the UAV (receiver) and the GS (transmitter) are shown in Fig. 2 and Fig. 3 for transmission frequencies of $28 \mathrm{GHz}$ and and $60 \mathrm{GHz}$, respectively. A common observation for RSS in all the scenarios is the two ray propagation model. The presence of scatterers introduces fluctuations in the received power. In case of sub-urban area, the fluctuations result in deviation from the two ray propagation model. At UAV height of $2 \mathrm{~m}$ above ground, the two ray propagation model holds only for a specific distance called the critical distance, or first Fresnel zone; after that the received power drops proportionally to 

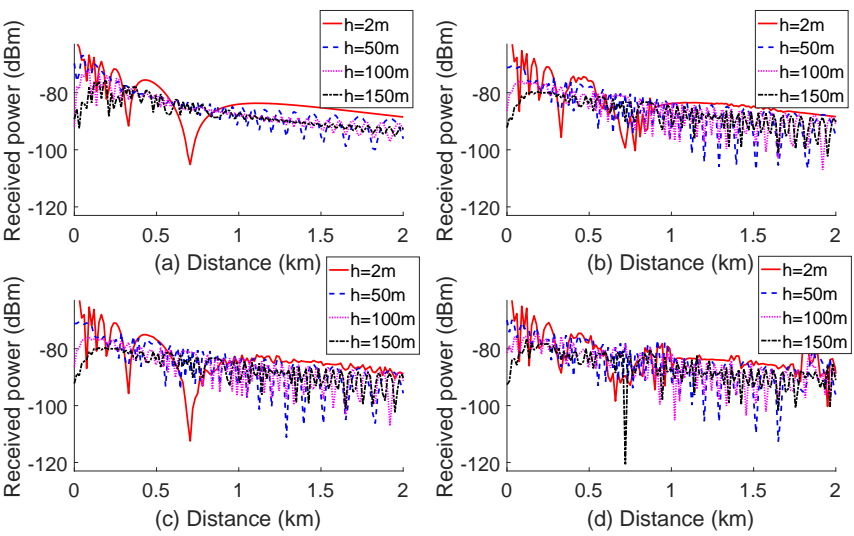

Fig. 2. Received power versus distance for different UAV heights at $28 \mathrm{GHz}$ in different scenarios: (a) Over sea, (b) Rural, (c) Suburban, (d) Urban.
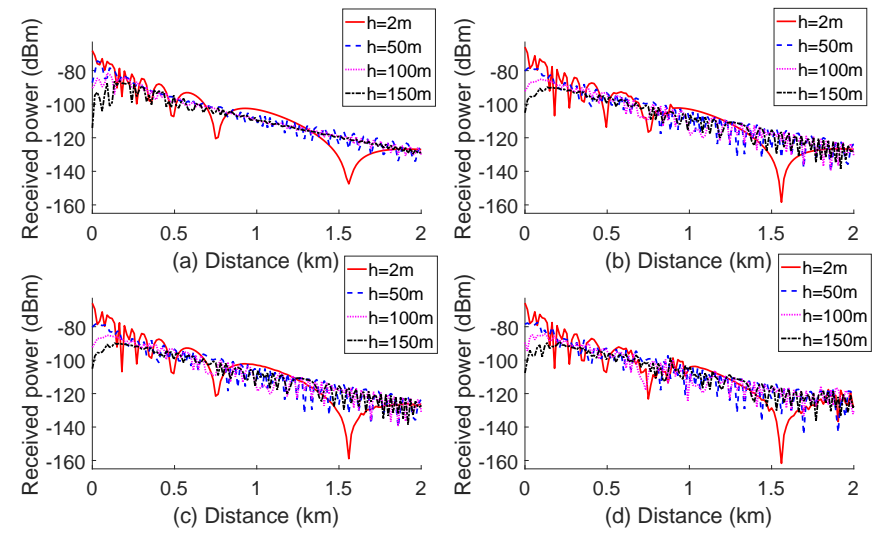

Fig. 3. Received power versus distance for different UAV heights at $60 \mathrm{GHz}$ in different scenarios: (a) Over sea, (b) Rural, (c) Suburban, (d) Urban.

some power of the distance [15]. The critical distance for $28 \mathrm{GHz}$ and $60 \mathrm{GHz}$ is different due to different RSS decay rates with respect to the distance.

Results in Fig. 2 and Fig. 3 show that as the height of the UAV increases, the maxima/minima appearance rate of the two ray propagation model increases as well. Moreover, the maxima/minima appearance rate is higher for $60 \mathrm{GHz}$ compared to $28 \mathrm{GHz}$. This is due to the smaller wavelength at $60 \mathrm{GHz}$ resulting in constructive and destructive interference more rapidly compared to $28 \mathrm{GHz}$. Additionally, the rate of RSS decay with respect to distance at $60 \mathrm{GHz}$ is higher than at $28 \mathrm{GHz}$ indicating higher path loss slopes as compared to $28 \mathrm{GHz}$. The RSS at $60 \mathrm{GHz}$ is lower compared to $28 \mathrm{GHz}$ as expected, due to higher losses at higher center frequencies.

The selection of relatively low heights in the simulations is due to regulations by the FAA [16] and this limits the effect of the UAV height on the RSS due to scatterers. If the height of the UAV is increased further, the effect of the scatterers on the RSS can be reduced.

In case of over sea scenario, the RSS closely follows the two ray propagation model with minimal fluctuations due to negligible effect of scatterers. On the other hand, in case of

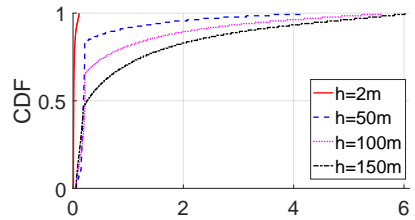

(a) RMS DS (ns)

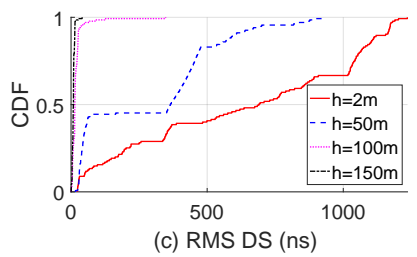

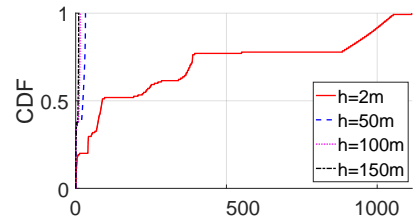

(b) RMS DS (ns)

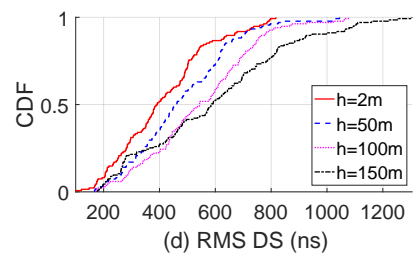

Fig. 4. CDF of RMS-DS for different UAV heights at $28 \mathrm{GHz}$ in different scenarios: (a) Over sea, (b) Rural, (c) Suburban, (d) Urban.

rural and suburban scenarios, we observe fluctuations in the RSS near the scatterers. These fluctuations become higher for urban areas due to large number of scatterers as shown in Fig. 2 and Fig. 3 An interesting effect is the shadowing of the scatterer reflections, due to the foliage of comparable size to the scatterers weakening the MPCs from scatterers. This can reduce the RSS fluctuations due to weaker reflected MPCs.

\section{Ray Tracing Results for the RMS-DS}

In Fig. 4 and Fig. 5. we present the cumulative distribution functions (CDFs) of the RMS-DS of the multipath channel between the GS transmitter and the UAV for four different environments, considering mmWave frequencies of $28 \mathrm{GHz}$ and $60 \mathrm{GHz}$, respectively. The RMS-DS results in Fig. 4 for the mmWave frequency of $28 \mathrm{GHz}$ show that the RMS-DS is the largest for urban environment for most of the UAV heights, due to high density of scatterers in the urban setting. Moreover, we observe that the RMS-DS increases as a function of the UAV height in the urban environment, for the trajectory shown in Fig. 1 b). The main reason for this behavior is that, at higher UAV altitudes, the UAV moves above the tall buildings, and it can observe signals that are scattered from larger number of surrounding buildings.

On the other hand, results in Fig. $4(\mathrm{~b})$ and $4(\mathrm{c})$ for rural and suburban environments show that, as opposed to the urban environment, the RMS-DS actually decreases with UAV altitude. In rural/suburban environments, the buildings are not as tall as the urban environment, and more sparsely deployed. Therefore, at higher UAV altitudes, the signals scattered from the buildings do not arrive at the UAV, hence reducing the RMS-DS. These different behavior of the multipath channel suggest that environmental factors and UAV height can have significant impact on the channel behavior and hence the receiver design.

The relative impact of different UAV heights on the RMSDS behavior observed to be similar in Fig. $5(60 \mathrm{GHz}$ mmWave band) when compared with the results in Fig. 4 for the $28 \mathrm{GHz}$ band. On the other hand, comparing the results in 


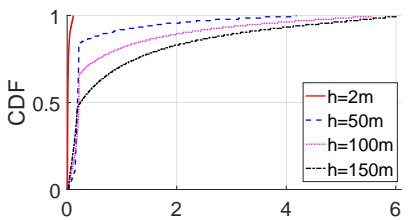

(a) RMS DS (ns)
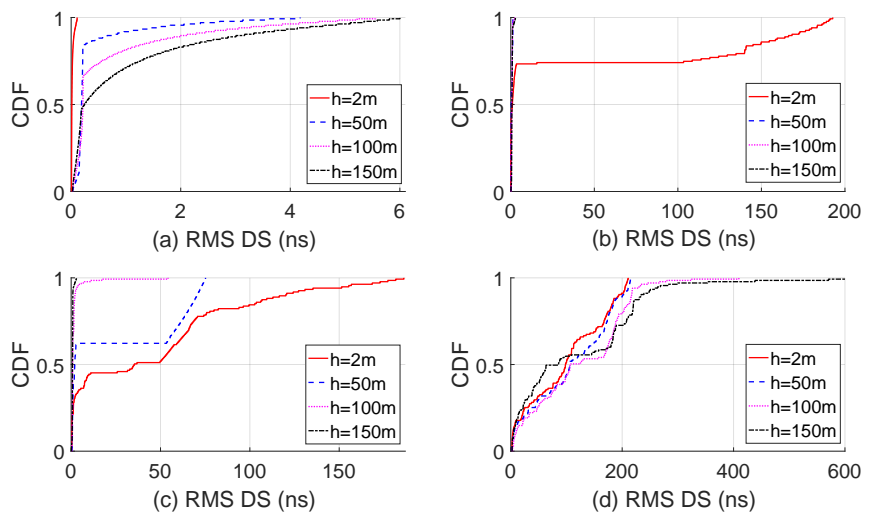

Fig. 5. CDF of RMS-DS for different UAV heights at $60 \mathrm{GHz}$ in different scenarios: (a) Over sea, (b) Rural, (c) Suburban, (d) Urban.

Fig. 4 and Fig. 5 with each other, RMS-DS is lower for the $60 \mathrm{GHz}$ band due to larger path loss at the $60 \mathrm{GHz}$ band.

\section{Channel SoUnding FOR MMWAVE UAVs}

While ray tracing simulations can provide interesting insights on the behavior of AG mmWave channels as discussed in Section III, propagation measurements at mmWave bands can more accurately characterize the subtle AG mmWave propagation features. For example, omni-directional RMS-DS with ray tracing simulations in [17] for an urban cellular environment have been found be relatively smaller when compared to the RMS-DS for mmWave propagation measurements in similar environments. However, developing a mmWave channel sounder for AG scenarios is a challenging task. Since the available UAVs have limited payload capacity, the basic requirement of a mmWave channel sounder is to be lightweight and compact. In this section, we provide a preliminary framework for AG channel sounding for mmWave UAV channels.

\section{A. USRP-Based Experimental Setup}

We use $60 \mathrm{GHz}$ Transmit/Receive (TX/RX) development system PEM009-KIT from Pasternack [18]. This kit accepts differential baseband I/Q signal as an input at the transmitter. The baseband single-ended I/Q signal is generated by using USRP X310 with LFTX daughterboard. This single ended I/Q stream is translated into differential I/Q stream using two $180^{\circ}$ ZFSCJ-2-1-S+ splitters from minicircuits. At the receiving end differential output from the PEM009-KIT is translated into single ended I/Q stream using two ZFSCJ-2-1-S+ splitters as combiners, which is then fed into USRP X310 receiver. The block diagram of the experimental setup is shown in Fig. 6.

In order to sound the channel the GNU-radio flow graph shown in Fig. 7 is used at the transmitter side. In this flow graph GLFSR source produces a maximal-length Pseudo Noise (PN) sequence $s[n] \in\{ \pm 1\}$ of degree 12, and of length $2^{12}-1=4095$. We define the sequence $\tilde{s}[n]$ as the periodic extension of $s[n]$ obtained by repeating the same sequence
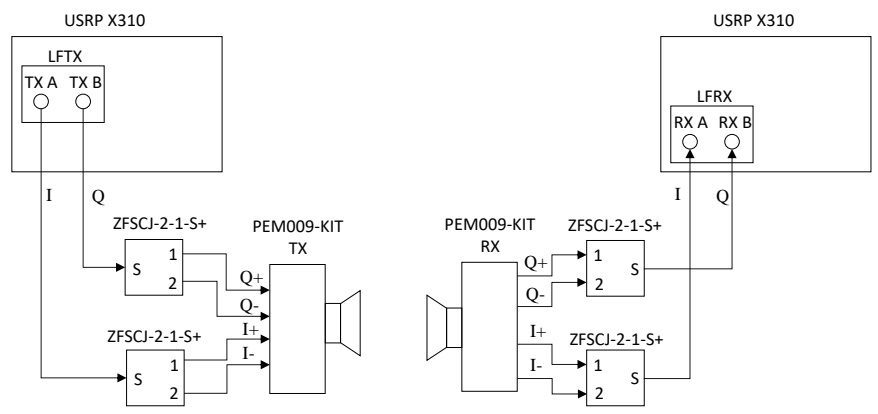

Fig. 6. The experimental channel sounder setup.

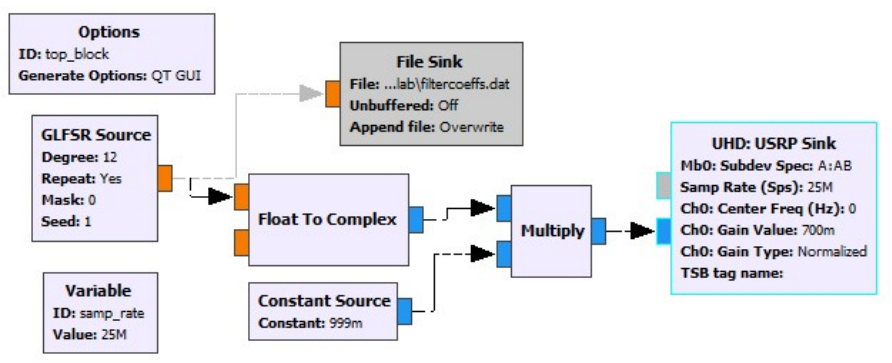

Fig. 7. The transmitter GNU radio flow graph for the channel sounder.

indefinitely. This signal is transmitted by the USRP with a sampling rate of $25 \mathrm{MHz}$ after being multiplied by a constant.

At the receiver side, the USRP source in the flow graph shown in Fig. 8 provides samples at $f_{\mathrm{s}}=25 \mathrm{MHz}$ sampling rate. The carrier frequency offset (CFO) between the transmitter and the receiver is estimated by first taking the square of the received signal. Since the transmitted signal is BPSK modulated, the spectrum of the squared signal contains a dominant peak at two times the value of the CFO. This estimate for the CFO is then used to compensate for the CFO in the received signal $y(n)$. By ignoring the noise, the received signal $y(n)$ can be expressed as the result of the signal $\tilde{s}(n)$ passing through the wireless multipath propagation channel of $N_{\mathrm{P}}$ MPCs as follows [19]

$$
y(n)=\sum_{p=0}^{N_{P}-1} a_{p} \exp \left(j \theta_{p}\right) \tilde{s}\left(n-\tau_{p}\right) .
$$

Since $s[n]$ can be assumed known at the receiver side, a matched filter, $g(n)=s(-n)$, can be applied after CFO correction to obtain

$$
\begin{aligned}
r(n) & =y(n) * g(n) \\
& =\sum_{p=0}^{N_{\mathrm{P}}-1} a_{p} \exp \left(j \theta_{p}\right) \tilde{R}_{s}\left(n-\tau_{p}\right),
\end{aligned}
$$

where $\tilde{R}_{s}(n)$ is periodic extension of deterministic autocorrelation function of $s(n)$. The signal $r(n)$ is composed of $N_{\mathrm{P}}$ attenuated, phase shifted, and delayed copies of the autocorrelation function, and it represents the channel impulse response (CIR) for a given environment. 


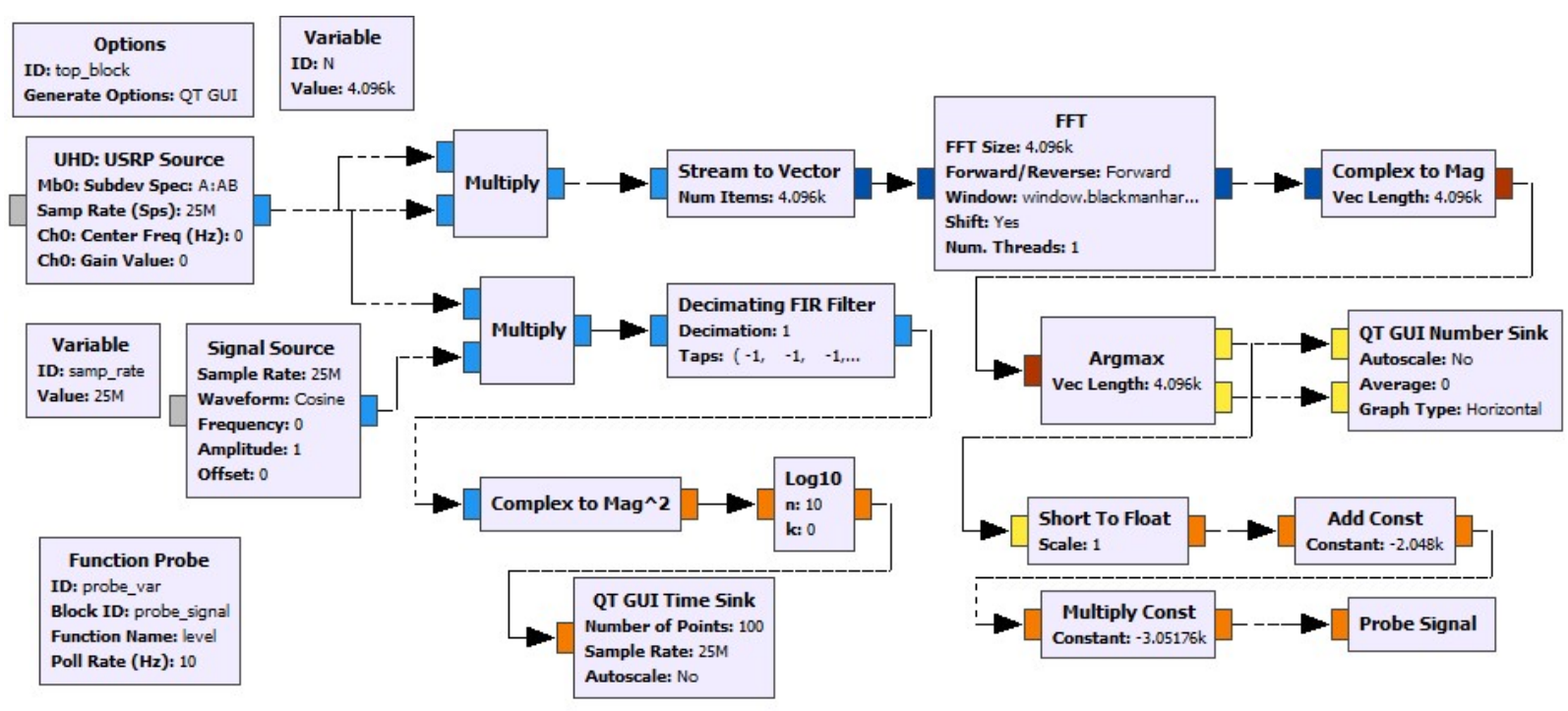

Fig. 8. The receiver GNU radio flow graph for the channel sounder.

\section{CONCLUSIONS}

In this paper, we provide ray tracing simulations for mmWave UAV AG propagation channels. It is observed that the two ray propagation model can be applicable (with some limitations) for urban, suburban, rural, and over sea scenarios. The presence of scatterers affect the RSS especially in case of urban scenario. The fluctuation rate of the RSS with respect to distance at $60 \mathrm{GHz}$ is higher when compared with $28 \mathrm{GHz}$. The RMS-DS behavior is highly dependent on the height of the UAV as well as the density/height of the scatters around the UAV. For the suburban and rural scenarios, we observe a lower RMS-DS when the UAV height increases, due to less significant effect of the scatterers at higher UAV heights. We also provide an experimental setup for AG mmWave channel sounding measurements using USRPs and GNU radio. The USRP-based channel sounder is lightweight enough to be carried, for example, at a DJI S-1000 octocopter, for conducting channel sounding experiments at $60 \mathrm{GHz}$ spectrum.

\section{REFERENCES}

[1] NTT DOCOMO Inc. and Ericsson, "New SID on Study on enhanced LTE Support for Aerial Vehicles," 3GPP Study Item Description (RP170742), Dubrovnik, Croatia, Mar. 2017.

[2] Qualcomm, "Qualcomm Technologies releases LTE drone trial results," Technical Report, May 2017, accessed: 2017-05-28. [Online]. Available: https://www.qualcomm.com/news/onq/2017/05/03/ qualcomm-technologies-releases-lte-drone-trial-results

[3] N. Rupasinghe, A. S. Ibrahim, and I. Guvenc, "Optimum hovering locations with angular domain user separation for cooperative UAV networks," in Proc. IEEE Global Telecommun. Conf. (GLOBECOM), 2016, pp. 1-6.

[4] M. Chen, M. Mozaffari, W. Saad, C. Yin, M. Debbah, and C. S. Hong, "Caching in the sky: Proactive deployment of cache-enabled unmanned aerial vehicles for optimized quality-of-experience," IEEE J. Select. Areas Commun. (JSAC), vol. 35, no. 5, pp. 1046-1061, 2017.

[5] A. Merwaday, A. Tuncer, A. Kumbhar, and I. Guvenc, "Improved throughput coverage in natural disasters: Unmanned aerial base stations for public-safety communications," IEEE Vehic. Technol. Mag., vol. 11, no. 4, pp. 53-60, 2016.
[6] FCC, "Use of Spectrum Bands Above $24 \mathrm{GHz}$ For Mobile Radio Services," July 2016, accessed: 2017-06-25. [Online]. Available: https://www.fcc.gov/document/spectrum-frontiers-ro-and-fnprm

[7] FCC, "Unlicensed Operation in the $57-64 \mathrm{GHz}$ Band," accessed: 2017-05-31. [Online]. Available: https://www.fcc.gov/document/ part-15-rules-unlicensed-operation-57-64-ghz-band

[8] M. R. Akdeniz, Y. Liu, M. K. Samimi, S. Sun, S. Rangan, T. S. Rappaport, and E. Erkip, "Millimeter wave channel modeling and cellular capacity evaluation," IEEE J. Select. Areas Commun., vol. 32, no. 6, pp. 1164-1179, 2014.

[9] S. K. Yoo, S. L. Cotton, R. W. Heath, and Y. J. Chun, "Measurements of the $60 \mathrm{GHz}$ UE to eNB Channel for Small Cell Deployments," IEEE Wireless Commun. Letters, vol. 6, no. 2, pp. 178-181, Apr. 2017

[10] C. A. L. Diakhate, J. M. Conrat, J. C. Cousin, and A. Sibille, "Millimeter-wave outdoor-to-indoor channel measurements at 3, 10, 17 and $60 \mathrm{GHz}$," in Proc. European Conf. Ant. Propag. (EUCAP), Mar. 2017, pp. 1798-1802.

[11] S. Salous and Y. Gao, "Wideband measurements in indoor and outdoor environments in the $30 \mathrm{GHz}$ and $60 \mathrm{GHz}$ bands," in Proc. European Conf. Ant. Propag. (EuCAP), Apr. 2016, pp. 1-3.

[12] W. Khawaja, I. Guvenc, and D. Matolak, "UWB channel sounding and modeling for UAV air-to-ground propagation channels," in Proc. IEEE Global Commun. Conf. (GLOBECOM), Dec. 2016, pp. 1-7.

[13] D. W. Matolak and R. Sun, "Air-ground channel characterization for unmanned aircraft systems-Part I: Methods, measurements, and models for over-water settings," IEEE Trans. Vehic. Technol., vol. 66, no. 1, pp. 26-44, 2017.

[14] R. Sun and D. W. Matolak, "Air-ground channel characterization for unmanned aircraft systems Part II: Hilly and mountainous settings," IEEE Trans. Vehic. Technol., vol. 66, no. 3, pp. 1913-1925, 2017.

[15] M. Elsayed, "Two-ray model in wireless communications," Technical Report, Apr. 2013, accessed: 2017-06-26. [Online]. Available: http://wireless-communications-systems.blogspot.com/2013/ 04/two-ray-model-in-wireless-communications.html

[16] FAA, "FAA rules for UAVs," accessed: 2017-02-25. [Online]. Available: https://www.faa.gov/uas/beyond_the_basics/

[17] Z. Zhang, J. Ryu, S. Subramanian, and A. Sampath, "Coverage and channel characteristics of millimeter wave band using ray tracing," in Proc. IEEE Int. Conf. Commun. (ICC), 2015, pp. 1380-1385.

[18] Pasternack, "60 GHz Development System," accessed: 2017-05-25. [Online]. Available: https://www.pasternack.com/ 60-ghz-development-system-low-phase-noise-pem009-kit-p.aspx

[19] J. M. Albornoz, "A Wideband Channel Sounder," MS Thesis, 2001, accessed: 2017-05-20. [Online]. Available: http://www2.ece.ohio-state. edu/ schniter/pdf/albornoz_thesis.pdf 